\title{
MEAN FLOW EFFECTS IN THE FARADAY INSTABILITY
}

\author{
ELENA MARTÍN \\ Depto. Ingeniería Mecánica, Máquinas y Motores Térmicos y Fluidos \\ E.T.S.I. Industriales, Universidad de Vigo \\ 36200 Vigo, Spain \\ CARLOS MARTEL and JOSÉ M. VEGA \\ Depto. Fundamentos Matemáticos \\ E.T.S.I. Aeronáuticos, Universidad Politécnica de Madrid \\ 28040 Madrid, Spain
}

Received 8 August 2002

\begin{abstract}
We study the weakly nonlinear evolution of Faraday waves in a $2 \mathrm{D}$ container that is vertically vibrated. In the small viscosity limit, the evolution of the surface waves is coupled to a non-oscillatory mean flow that develops in the bulk of the container. The corresponding long time (Navier-Stokes+amplitude) equations are derived and analyzed numerically. The results indicate that the (usually ignored) mean flow plays an essential role in the stability of the surface waves and in the bifurcated wave patterns.
\end{abstract}

\section{Introduction}

The Faraday ${ }^{1}$ instability, i.e. the parametric excitation of waves at the free surface of a liquid by vertical vibration, has attracted a great deal of attention because of the rich variety of nonlinear pattern-forming phenomena that exhibits (see e.g. Refs. 2-4 and references therein). Yet, despite of much study, certain fundamental questions still have not been successfully answered. One such questions is the correct description of the weakly nonlinear regime near the onset of Faraday instability in the singular limit of small viscosity. The nonlinear amplitude equations that are often used in the literature are obtained from a strictly inviscid formulation and corrected a posteriori by adding some linear dissipation terms. ${ }^{3,5}$ This formulation ignores the slow mean flow that is driven by the boundary layers at the container walls and free surface and, in the case of a monochromatic wave, predicts standing waves (SW) as the only attractor after onset and fails to reproduce the drifting SW that have been observed experimentally. ${ }^{6,7}$ In this paper we shall briefly outline the derivation of a system of equations for the slow time evolution of the surface waves and the mean flow, starting from the full Navier-Stokes equations. In addition, we shall present some examples of the new states that are obtained (see Ref. 11 for a more complete analysis). 


\section{Coupled Amplitude-Mean Flow Equations}

We consider a very simple configuration, namely a small 2-D, horizontally periodic layer of incompressible fluid (Fig. 1).

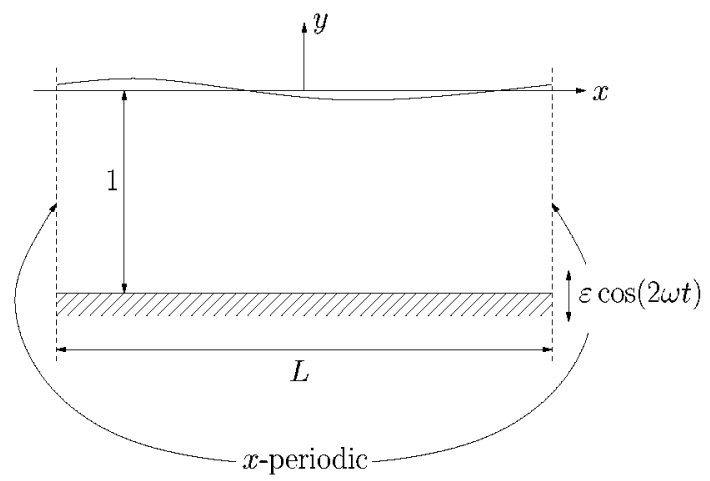

Fig. 1. Sketch of the two-dimensional fluid layer.

The governing equations, made nondimensional with the height of the unperturbed free surface and the gravitational time, are

$$
\begin{gathered}
u_{x}+v_{y}=0, \\
u_{t}+v\left(u_{y}-v_{x}\right)=-q_{x}+C\left(u_{x x}+u_{y y}\right), \\
v_{t}-u\left(u_{y}-v_{x}\right)=-q_{y}+C\left(v_{x x}+v_{y y}\right), \\
u=v=0 \quad \text { at } y=-1, \\
v=f_{t}+u f_{x}, \quad\left(u_{y}+v_{x}\right)\left(1-f_{x}^{2}\right)+2\left(v_{y}-u_{x}\right) f_{x}=0 \quad \text { at } y=f, \\
q-\left(u^{2}+v^{2}\right) / 2+4 \omega^{2} \varepsilon f \cos (2 \omega t)-f+T f_{x x} /\left(1+f_{x}^{2}\right)^{3 / 2} \\
=2 C\left[v_{y}+u_{x} f_{x}^{2}-\left(u_{y}+v_{x}\right) f_{x}\right] /\left(1+f_{x}^{2}\right), \quad \text { at } y=f,
\end{gathered}
$$

$$
u, v, q \text { and } f \text { are periodic, of period } L \text {, in } x \text {. }
$$

Here $u$ and $v$ are the horizontal and vertical velocity components, $f$ is the free surface elevation (measured from the undisturbed position), and

$$
q=\text { pressure }+\left(u^{2}+v^{2}\right) / 2+y-4 \omega^{2} \varepsilon y \cos (2 \omega t) ;
$$

$2 \omega$ and $\varepsilon$ are the forcing frequency and amplitude, $C=\nu /\left(g h^{3}\right)^{1 / 2}(\nu=$ kinematic viscosity) is a ratio of viscous to gravitational effects and $T^{-1}=\rho g h^{2} / \sigma$ ( $\rho=$ density, $\sigma=$ surface tension) is the Bond number.

In the following we shall derive equations for the weakly nonlinear evolution of small, nearly-resonant solutions at small viscosity, i.e.

$$
|u|+|v|+|q|+|f| \ll 1, \quad \varepsilon \ll 1, \quad\left|\omega-\omega_{0}\right| \ll 1, \quad C \ll 1,
$$


where $\omega_{0}$ is a natural oscillation frequency of the system in the inviscid $(C=0)$ limit. There are two kinds of nearly marginal modes ${ }^{10}$ of the unforced linearized version of (1)-(7): (i) inviscid modes, that produce surface waves, with a $\mathcal{O}(1)$ frequency and a $\mathcal{O}(\sqrt{C})$ decay rate, and (ii) viscous modes, with a very small $\mathcal{O}(C)$ decay rate, that produce a mean flow and do not deform the free-surface at first order.

Taking into account all the above mentioned modes, the solution can be expanded as

$$
\begin{aligned}
& u=U_{0}(y) e^{i \omega t}\left[A(t) e^{i k x}-B(t) e^{-i k x}\right]+c . c .+u^{s}(x, y, t)+\cdots, \\
& v=V_{0}(y) e^{i \omega t}\left[A(t) e^{i k x}+B(t) e^{-i k x}\right]+c . c .+v^{s}(x, y, t)+\cdots, \\
& q=Q_{0}(y) e^{i \omega t}\left[A(t) e^{i k x}+B(t) e^{-i k x}\right]+c . c .+q^{s}(x, y, t)+\cdots \\
& f=e^{i \omega t}\left[A(t) e^{i k x}+B(t) e^{-i k x}\right]+c . c .+f^{s}(x, t)+\cdots
\end{aligned}
$$

where c.c. stands for the complex conjugate, $k=2 m \pi / L$ with $m=$ integer is the horizontal wavenumber with frequency closest to $\omega$, and $U_{0}, V_{0}$ and $Q_{0}$ are the corresponding inviscid eigenfunctions. The terms displayed above correspond to the only surface mode that is (sub-harmonically) excited by the external forcing and the streaming flow that will be denoted hereinafter by the superscript $s$. Dependence of the amplitudes $A$ and $B$ on $x$ is ignored for simplicity, see Refs. 8 and 9 for a more complicated analysis including spatial wave modulations.

If we now insert the expansions (9)-(12) into the governing equations, take into account the boundary layers at the free surface and the bottom of the container and apply solvability conditions, the following equations are obtained

$$
\begin{gathered}
A^{\prime}=\left[-\delta-i d+\mathrm{i} \alpha_{3}|A|^{2}-\mathrm{i} \alpha_{4}|B|^{2}-\mathrm{i} \alpha_{6} \frac{1}{L} \int_{-1}^{0} \int_{0}^{L} g(y) u^{s} d x d y\right] A+\mathrm{i} \varepsilon \alpha_{5} \bar{B}, \\
B^{\prime}=\left[-\delta-i d+\mathrm{i} \alpha_{3}|B|^{2}-\mathrm{i} \alpha_{4}|A|^{2}+\mathrm{i} \alpha_{6} \frac{1}{L} \int_{-1}^{0} \int_{0}^{L} g(y) u^{s} d x d y\right] B+\mathrm{i} \varepsilon \alpha_{5} \bar{A}, \\
u_{x}^{s}+v_{x}^{s}=0, \\
u_{t}^{s}+v^{s}\left(u_{y}^{s}-v_{x}^{s}\right)=-\tilde{q}_{x}^{s}+C\left(u_{x x}^{s}+u_{y y}^{s}\right), \\
v_{t}^{s}-\left[u^{s}+\left(|B|^{2}-|A|^{2}\right) g(y)\right]\left(u_{y}^{s}-v_{x}^{s}\right)=-\tilde{q}_{y}^{s}+C\left(v_{x x}^{s}+v_{y y}^{s}\right), \\
u^{s}=\alpha_{7}\left[i A \bar{B} e^{2 i k x}+\text { c.c. }+|B|^{2}-|A|^{2}\right], \quad v^{s}=0 \quad \text { at } y=-1, \\
\partial u^{s} / \partial y=\alpha_{8}\left(|B|^{2}-|A|^{2}\right), \quad v^{s}=0 \quad \text { at } y=0, \\
u^{s}, v^{s} \text { and } \tilde{q}^{s} \text { are periodic, of period } L, \text { in } x .
\end{gathered}
$$

These equations describe the coupled evolution of the wave amplitudes $(A, B)$ and the streaming flow $\left(u^{s}, v^{s}, q^{s}, f^{s}\right)$ in the slow time scale (see Ref. 11 for a more detailed derivation of the equations above and for the expressions of the coefficients 
and the function $g(y)$ ). Notice that the mean flow is not a simple by-product of the waves, but it affects the dynamics of the surface waves through the averaged terms in (13) and (14), which represent a transport of the waves by the mean flow.

\section{Results}

The solution of equations (13) and (14) always relaxes to a standing wave $(|A|=$ $\left.|B|=R_{0}\right)$ of the form

$$
f(x, t)=4 R_{0} \cos \left(\omega t+\phi_{0}\right) \cos [k(x-\psi)],
$$

with constant amplitude $R_{0}$ (which depends on the amplitude of the applied forcing) and spatial phase $\psi(t)$ that remains coupled to the streaming flow through the equation

$$
d \psi / d t=\left[\alpha_{6} /(k L)\right] \int_{-1}^{0} \int_{0}^{L} g(y) u^{s}(x, y, t) d x d y .
$$

The system of equations (22) and (15)-(20) has been solved numerically for different values of the parameters: the streaming flow Reynolds number

$$
R e=6 R_{0}^{2} \omega_{0} k /\left(C \sinh ^{2} k\right),
$$

the wave number $k$, and the spatial period $L=2 m \pi / k$. The typical solution that sets in for small Re (small forcing) is represented in Fig. 2a and corresponds to two identical pairs of steady counterrotating eddies below the steady SW (the vertical lines in Figs. 2 and 3 corresponds to the nodes of the SW). Since this steady solution

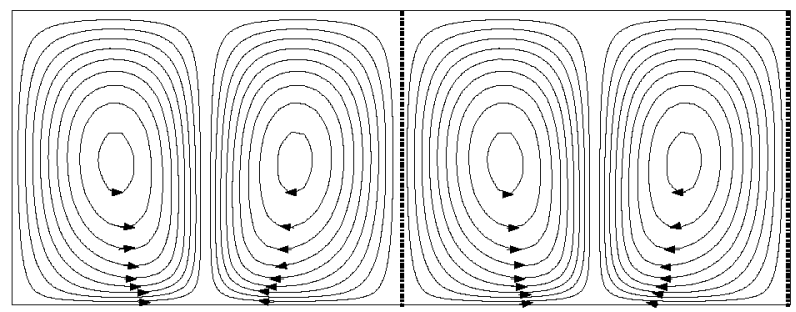

(a) $R e=260$

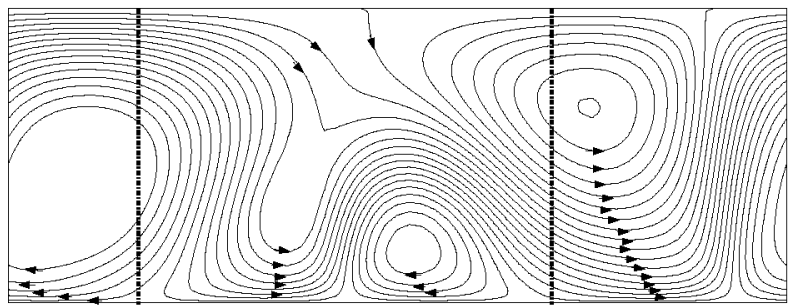

(b) $R e=325$

Fig. 2. Streamlines of the streaming flow for $k=2.37, m=1$ and (a) $R e=260$ and (b) $R e=325$ (in moving axes). Thick vertical lines correspond to the nodes of the surface waves given by (21). 
(a)
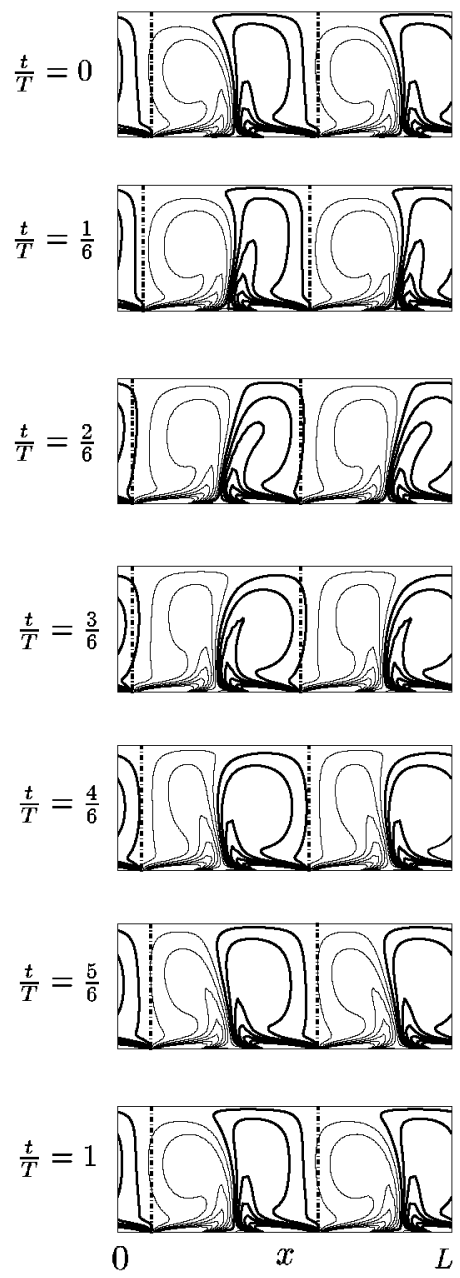

(b)
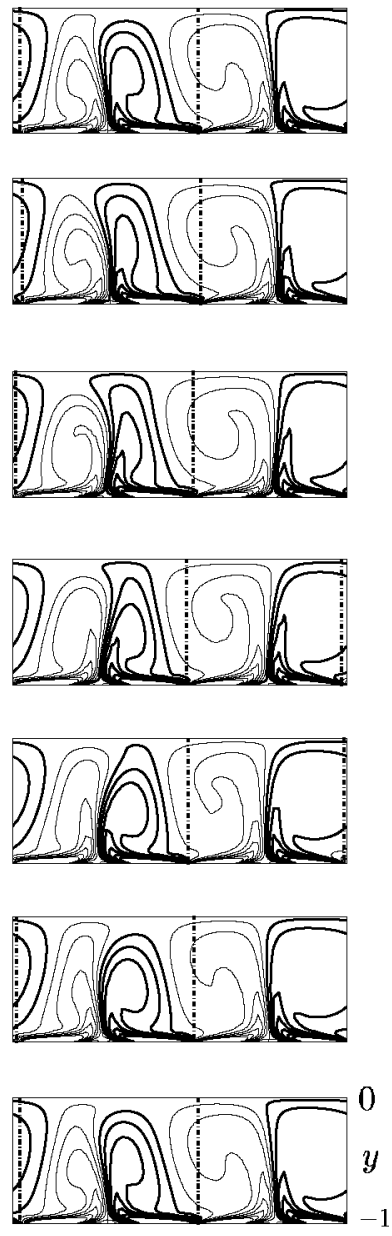

Fig. 3. Constant vorticity contours for the streaming flow (thick: positive, thin: negative) for two periodic solutions for $k=2.37, m=1$ and (a) $R e=290$ and (b) $R e=400$. Vertical lines as in Fig. 2.

is reflection symmetric in $x$, the integral in (22) vanishes and the streaming flow does not affect the surface SW. If Re exceeds a threshold value this steady solution becomes unstable through a Hopf bifurcation and a family of time periodic solutions appears. One of these periodic solutions is represented in Fig. 3a. Notice that the streaming flow is no longer reflection symmetric and the integral in (22) is nonzero and produces a time periodic drift of the SW on the free surface. These periodic solutions resemble the so called "compression modes" that have been observed in experiments in annular containers ${ }^{6,7}$ and cannot be obtained with the usual amplitude equations that ignore the coupling with the streaming flow. For higher 
values of $R e$ the $L / 2$ periodicity is lost (see Fig. $3 \mathrm{~b}$ ) and, for still higher Re, the system jumps to a branch of solutions with constant drift velocity $\left(\psi^{\prime}=c\right)$ like the one plotted in Fig. 2b, which is steady in a reference frame that moves with the drift velocity (these states have also been observed experimentally). ${ }^{6,7}$ For larger values of $L$ several different solutions coexist and some of them exhibit complex behavior in time. ${ }^{11}$ For all these states, which are not steady $\mathrm{SW}$, the coupling with the streaming flow is an essential ingredient that should not be ignored.

\section{Acknowledgments}

This work was supported by the Spanish DGI under grant BFM2001-2363 and by NASA under grant NAG3-2152.

\section{References}

1. M. Faraday, Phil. Trans. Roy. Soc. London 121, 319 (1831).

2. A. B. Ezerskii, P. I. Korotin and M. I Rabinovich, JETP Lett. 41, 157 (1985).

3. J. Miles and D. Henderson, Annu. Rev. Fluid Mech. 22, 143 (1990).

4. A. Kudrolli and J. P. Gollub, Physica D97, 133 (1997).

5. M. Cross and P. C. Hohenberg, Rev. Mod. Phys. 65 3, 851 (1993).

6. S. Douady, S. Fauve and O. Thual, Europhys. Lett. 10, 309 (1989).

7. O. Thual, S. Douady and S. Fauve, in Instabilities and Nonequilibrium Structures II, ed. E. Tirapegui and D. Villaroel (Kluwer, 1989), p. 227.

8. J. M. Vega, E. Knobloch and C. Martel, Physica D154, 313 (2001).

9. V. Lapuerta, C. Martel and J. M. Vega, Physica D, in press (2002).

10. C. Martel and E. Knobloch, Phys. Rev. E56, 5544 (1997).

11. E. Martín, C. Martel and J. M. Vega, J. Fluid Mech., in press (2002). 